\title{
Dissociative Symptoms and Self-Reported Childhood and Current Trauma in Male Incarcerated People with Borderline Personality Disorder - Results from a Small Cross-Sectional Study in Iran
}

This article was published in the following Dove Press journal:

Neuropsychiatric Disease and Treatment

\section{Sanobar Golshani ${ }^{1, *}$ Sahel Ghanbari ${ }^{1} *$ \\ Ali Firoozabadi ${ }^{2}$ Jalal Shakeri' \\ Sarah Hookari ${ }^{1}$ \\ Bahareh Rahami' \\ Dena Sadeghi Bahmani $\mathbb{D}^{1,3,4}$ \\ Serge Brand (iD) $1,3-6$ \\ 'Substance Abuse Prevention Research Center, Health Institute, Kermanshah University of Medical Sciences, Kermanshah, Iran; ${ }^{2}$ Research Center for Psychiatry and Behavioral Sciences, Department of Psychiatry, Hafez Hospital, Shiraz University of Medical Sciences, Shiraz, Iran; ${ }^{3}$ Sleep Disorders Research Center, Kermanshah University of Medical Sciences, Kermanshah, Iran; ${ }^{4}$ University of Basel, Psychiatric Clinics (UPK), Center for Affective-, Stress- and Sleep Disorders (ZASS), Basel 4002, Switzerland; ${ }^{5}$ Department of Sport, Exercise, and Health, Division of Sport Science and Psychosocial Health, University of Basel, Basel, Switzerland; ${ }^{6}$ Department of Psychiatry, School of Medicine, Tehran University of Medical Sciences, Tehran, Iran \\ *These authors contributed equally to this work}

Correspondence: Serge Brand University of Basel, Psychiatric Clinics (UPK), Center for Affective-, Stress- and Sleep Disorders (ZASS), Basel 4002,

Switzerland

$\mathrm{Tel}+41613255097$

Email serge.brand@unibas.ch
Background: There is evidence that incarcerated people show higher rates of symptoms of psychopathology. In the present study, we assessed male Iranian incarcerated people with borderline personality disorders (BPD) and investigated the occurrence of past and current trauma and their associations with dissociative experiences.

Methods: A total of 69 male Iranian incarcerated people (mean age: 33.76 years) diagnosed with PBD completed questionnaires covering sociodemographic information, dissociative experiences, and past and current traumatic events.

Results: Participants reporting the occurrence of childhood trauma also reported the occurrence of adulthood trauma. Dissociation and adulthood trauma were associated in a U-shaped, non-linear fashion: Low and high adulthood trauma were associated with higher dissociation. Younger age, the presence of childhood trauma, and being single or divorced predicted adulthood trauma.

Conclusion: The pattern of results suggests that both childhood and adulthood trauma are highly prevalent among male incarcerated people, while the association between adulthood trauma and dissociation appeared to be more complex. When treating male incarcerated people, a complex interplay between past and current traumas and dissociation should be considered.

Keywords: male incarcerated people, childhood trauma, adulthood trauma, dissociation, borderline personality disorders

\section{Introduction}

It is estimated that about nine million individuals ( $1 \%$ of the world population) are temporary or permanently imprisoned, and about 30 million individuals are released worldwide from prison every year. ${ }^{1}$

Numerous studies have shown that, compared to the general population, incarcerated people have higher rates of mental disorders. ${ }^{2-4}$ This holds true for instance for female incarcerated people in Canada, ${ }^{2}$ or for incarcerated people in low- and middle-income countries. ${ }^{3}$ Henry $^{5}$ analyzed data of $18^{\prime} 185$ incarcerated people in State and Federal Correctional Facilities in the USA and identified high rates of adverse childhood and adulthood experiences, mental health and substance use disorders. Further, Fazel et $\mathrm{al}^{4}$ reported in their systematic review of publications on female incarcerated people and older adult incarcerated people that mental 
health issues ranged from 3.6\% (psychotic illnesses) up to $60 \%$ (drug misuse). But Fazel, Hayes et al ${ }^{4}$ also mentioned that studies did not thoroughly report which diagnostic classifications were used, and who diagnosed incarcerated people's psychiatric disorders. Baranyi et $\mathrm{al}^{3}$ included in their systematic review (SR) and meta-analysis (MA) studies from low- and middle-income countries such as Brazil, India, Turkey, Egypt, Chile, South Africa, Sri Lanka, Nigeria, Burkina Faso, South Sudan, Iran, Togo, and Malaysia. To assess psychiatric disorders, either the DSM-III-R, the DSM-IV or the ICD 10 were used. Baranyi et $\mathrm{al}^{3}$ reported the following prevalence rates: psychosis: $6.2 \%$; major depressive disorders: $16.0 \%$; alcohol use disorder: $3.8 \%$; substance use disorder: $5.1 \%$. Further, and compared to the general population, Baranyi et $\mathrm{al}^{3}$ reported the following risk ratios among incarcerated people: non-affective psychosis: 16-fold higher risk; major depression and illicit drug use disorder: six-fold higher risk; alcohol use disorders: two-fold higher risk. Note that in most countries mentioned in Baranyi et $\mathrm{al}^{3}$ for religious reasons alcohol is prohibited; accordingly, the prevalence rates of alcohol misuse were lower, when compared to Western countries. Finally, Conn et $\mathrm{al}^{6}$ used self-ratings and experts' rating based on the DSM-IV and showed that among a sample of incarcerated people in the USA both personality disorder and substance use disorders appeared to be more frequent, when compared to the general population.

Reingle Gonzalez and Connell ${ }^{7}$ gathered data from $18^{\prime} 185$ incarcerated people in the US; to assess psychiatric disorders, participants reported whether they had been given a psychiatric diagnosis (based on the DSM-IV) by a mental health professional such as a psychiatrist or psychologist. Reingle Gonzalez and Connell ${ }^{7}$ found in their study that incarcerated people's mental health issues were not adequately addressed, and that a poorly adequate treatment of psychiatric issues was correlated with higher risks of recidivism and health care costs, once a prisoner was released.

Last, as regards suicidal behavior, Marzano et $\mathrm{al}^{8}$ and Valizadeh et $\mathrm{al}^{9}$ reported strong associations between psychiatric disorders and suicidal behavior among incarcerated people; specifically, psychiatric morbidity and comorbidities, and trauma, along with social isolation, increased the risk of suicidal behavior. ${ }^{8}$ Likewise, most recently, Favril et $\mathrm{al}^{10}$ showed in their SR and MA of 35 studies from 20 countries including 663'735 participants, that $3.8 \%$ of them reported self-harm-related behavior.
Among other factors, current psychiatric diagnoses, particularly major depressive disorders and borderline personality disorder (BPD), and experiencing sexual or physical victimization were important predictors of self-harm. Importantly, beside misuse of multiple substances, BPD was also a strong predictor of self-harm among a larger sample of incarcerated people reporting self-harming behavior. ${ }^{11}$

To summarize, studies from countries with different cultural, religious and economical background showed that psychiatric disorder is a general health concern among incarcerated people. Specifically, personality disorders are frequently observed; as a result, in the present study, we focused on male incarcerated people with borderline personality disorders (BPD).

To describe BPD, Leichsenring, Leibing, Kruse, New and Leweke ${ }^{12}$ reported in their overview that BPD is a common mental disorder characterized by a profound pattern of instability. This instability concerns communication and interpersonal relationships, identity, impulsivity, and affect. Typically, individuals with BPD show suicidal tendency, self-injury and unstable relationships. Individuals with BPD report disturbed relatedness, behavioral dysregulation, affective dysregulation, and chronic feelings of emptiness. Leichsenring et $\mathrm{al}^{12}$ further emphasized that in the general population the prevalence rates of BPD range from $0.5 \%$ to $5.9 \%$, while in clinical populations, BPD is the most common personality disorder (prevalence rate: about $10 \%$ ). Further, typically, individuals with BPD suffer from anxiety and depressive disorders, and substance misuse. The diagnose of post-traumatic stress disorder (PTSD) is often observed, but not universal in all individuals with BPD.

Last, as regards the etiology of BPD, genetic and environmental factors, and a gene-environment interaction appear to describe best the emergence of BPD. Specifically, a genetic susceptibility to BPD appears to be further enhanced by adverse childhood experiences such as physical and sexual maltreatment and psychological maltreatment such as psychological and social neglect.

As regards the situation of incarcerated people in Iran, there are currently more than 163,000 incarcerated people. Following Sepehrmanesh et al, ${ }^{13}$ the prevalence of psychiatric disorders among Iranian incarcerated people was $75 \%$ in Shiraz and $88 \%$ in Tehran, and with prevalence rates for personality disorders of up to $88 \%$ in the Sanandaj prison in Northwestern Iran. Note that in the study of Sepehrmanesh et al, ${ }^{13}$ trained psychiatrists and 
clinical psychologists diagnosed participants based on the DSM-5. Valizadeh et $\mathrm{al}^{9}$ screened publications on psychiatric disorders in Iranian incarcerated people. The key inclusion criterion for their SR and MA was that psychiatric and clinical interviews were based on the DSM-IV. Further, Valizadeh et $\mathrm{al}^{9}$ found that based on a thorough clinical interview $24 \%$ of incarcerated people suffered from antisocial personality disorders, and $7.1 \%$ of incarcerated people suffered from BPD. In this respect, several studies have shown that a thorough treatment of psychiatric disorders among incarcerated people was associated with current and future health and behavioral issues.

As regards psychiatric disorders in adulthood, previous work has shown that about half of all psychiatric issues emerge during childhood and early adolescence, ${ }^{14,15}$ and that these psychiatric issues continue into late adolescence and adulthood. For example, Mohr-Jensen and Steinhausen ${ }^{16}$ showed in their SR and MA that diagnosis of childhood attention-deficit/hyperactivity disorder was associated with a higher risk of antisocial behavior and incarceration in later life. Typically committed crimes were those related to theft, assault, drugs and weapons.

Additionally, childhood maltreatment is one of the main causes of psychiatric issues in adolescences and adulthood. ${ }^{17}$ Konkoly et $\mathrm{al}^{18}$ showed in their systematic review that exposure to interpersonal trauma during childhood increased the risk of substance use disorders (in particular alcohol abuse) in later life. Not surprising, childhood and adulthood adversity were important determinants of poor mental health and high prevalence rates of substance use disorders among a larger sample of incarcerated people. ${ }^{5}$

Further, dissociation often occurs as a coping response to psychological trauma when the individual's skills for emotion regulation are insufficient to control and monitor the emotional experience during and after the traumatic event. Following others, ${ }^{19-21}$ dimensions of dissociation are characterized by the disruption and/or discontinuity in the normal integration of consciousness, memory, identity, emotion, perception, body representation motor control, and behavior. Typically, a person with symptoms of dissociation reports unwanted disruptions of consciousness and behavior, accompanied by the loss of continuity of subjective experience. Dissociation is thus understood as a psychological process to cope with traumatic events, and is comprised of the following dimensions: depersonalization, derealization, time distortion, dissociative flashbacks, alterations in the perception of the self. Scalabrini et $\mathrm{al}^{22}$ reported that these dimensions are often observed in, but not limited to, individuals with borderline personality disorders (BPD). Likewise, Vermetten and Spiegel ${ }^{19}$ and Sar et $\mathrm{al}^{23}$ reported higher dissociation scores in individuals with spectrum BPD, again compared to healthy controls. Vermetten and Spiegel ${ }^{19}$ also reported that, compared to healthy controls, individuals with BPD had higher childhood trauma rates, while Tyrka et $\mathrm{al}^{24}$ emphasized that childhood maltreatment increases the risk of suffering from personality disorder symptoms, and not only BPD as such. Further, Leichsenring et $\mathrm{al}^{12}$ emphasized that genetic and environmental factors and a gene-environment interaction appear to best describe the emergence of BPD.

However, to our knowledge, evidence on the association between BPD and childhood trauma is not so far available for Iranian male incarcerated people. The first aim of the present study, therefore, was to investigate dissociation rates in a sample of male incarcerated people with BPD, while the second aim was to determine whether and if so to what extent dissociations were related to selfreported childhood or adulthood traumas.

For the following reasons, we believe that findings from this study may be of importance. First, mental disorders such as antisocial behavior and personality disorders are strongly associated with involvement in crime. Second, if BPD is related to childhood or current trauma, treatment options should take these traumatic experiences into account. Third, results from the study may have implications for treatment once an individual with BPD is released from prison. Last, to the best of our knowledge, no such studies on the associations between past and current trauma and dissociation have been performed so far in Iran; if this is the case, the present study might have the potential to add to our understanding of the association between childhood, adulthood trauma, and dissociative symptoms in a further way.

The following two hypotheses and one research question were formulated. First, following others, ${ }^{17,19}$ we expected that childhood trauma would be more often reported by male incarcerated people with BPD, compared to normative data. ${ }^{25}$ Second, following others, ${ }^{19,22,23}$ we expected an association between levels of dissociation and the prevalence rates of self-reported childhood and current/adulthood trauma. Third, we treated as an exploratory research question whether age, civil status, levels of dissociation and the prevalence rates of self-reported childhood could predict current/adulthood self-reported trauma. The exploratory research question is based on the 
assumption that childhood trauma and the degree of dissociation might be concomitantly and negatively associated with adulthood trauma, and that sociodemographic dimensions such as higher age, marital status as a proxy of social relationships, and educational levels as a proxy of more economic and cognitive resources might counterbalance the presence of adulthood trauma.

\section{Methods}

\section{Procedure}

Male incarcerated people of the central prison of Kermanshah (Kermanshah, Iran) were approached to participate in the present study. The central prison of Kermanshah serves the city of Kermanshah (about 1.1 million habitants) and the catchment area of Kermanshah Province (about 900'000 habitants). Eligible participants were fully informed about the aims of the study and the confidential data handling. Most importantly, to exclude participation due to misleading expectations, eligible participants were fully informed and assured that the participation or non-participation to the study neither had advantages nor disadvantages as regards the status of imprisonment and any kind of treatment. Thereafter, they signed a written informed consent. Next, participants completed a series of questionnaires covering sociodemographic information, retrospectively assessed childhood trauma, and current dimensions of dissociation and current trauma. The ethics committee of the Kermanshah University of Medical Sciences (KUMS, Kermanshah, Iran; KUMS.REC.1394.497) approved the study which was performed in accordance with rules laid down in the seventh and current form ${ }^{26}$ of the Declaration of Helsinki.

\section{Sample}

The sample consisted of 69 male incarcerated people. Inclusion criteria were: 1. Age between 18 and 65 years. 2 . Diagnosis of BPD, as ascertained by an experienced and trained clinical psychologist, and based on the SKID II clinical interview for DSM-IV personality disorders. ${ }^{27} 3$. Willing and able to comply with the study requirements. 4 . Signed written informed consent. Exclusion criteria were: 1. Current state of psychosis. 2. Current suicidality. 3. Axis-I psychiatric disorders, as ascertained by an experienced and trained clinical psychologist based on the DSM IV interview, ${ }^{28}$ except tobacco use disorder or cannabis use. 4. Current physical illness such as chronic pain, infectious diseases, allergies, and needing special medication with possible mood-, pain-, or memoryaltering effects. 5. Withdrawal from the study.

Note that being illiterate was not an exclusion criterion; in this case, a psychologist conducted an interview and assisted the participant in completing the questionnaires.

\section{Tools}

\section{Sociodemographic Information}

Participants reported on their age, marital status (single, married, divorced, widowed), highest educational level (illiterate and elementary school; guidance school (most modest level of mandatory secondary school) and high school; academic level), current job (yes vs no), living area (urban vs rural area), and type of crime committed.

\section{Dissociative Experiences Scale (DES)}

The Dissociative Experiences Scale (DES) ${ }^{29}$ is a selfreport questionnaire for dissociative experiences and consists of 28 items. Ghaffarinejad et al $^{30}$ reported satisfactory psychometric properties of the Persian version. A typical item is: "I have had this experience that I have felt that other people, other objects and the world around me are not real." Answers are given on 10-point rating scales with the anchor points $0 \%$ (= never) to $100 \%$ (= always); higher mean scores reflect a more pronounced experience of dissociation. The overall score ranges from $0 \%$ to $100 \%$. Additionally, the following categories were used: $0-15 \%$ : no dissociative experience; 15.1-20\%: some dissociative experiences; 20.1-30\%: elevated dissociative experiences; 30.1-40\%: clearly elevated dissociative experiences and suggestive of PTSD or Dissociative Disorder Non Otherwise Specified (DDNOS); 40.1 points or higher: high possibility of Dissociative Identity Disorder (DID), or increased likelihood of DID. The scores were also used as continuous variable, as evidence shows that the odds of having a dissociative disorder increases with higher scores.

\section{Traumatic Events}

The Traumatic Experience Checklist ${ }^{31,32}$ was employed to assess past and current traumatic events. It consists of 13 items; six items refer to traumatic experiences during childhood and adolescence (eg, "Sexual contact before you were 18 with somebody who was 5 or more years older than you"; "life-threatening illness"); seven items refer to recent or traumatic events during adulthood (eg, "imprisonment"; "torture"; "sexual assault by a family member or someone you know"). Answers are given in a forcedchoice dichotomous fashion (no $=0$; yes $=1$ ) and with 
higher sum scores reflecting a greater frequency of traumatic events.

\section{Statistical Analysis}

A one-sample $t$-test was computed to compare normative levels of childhood trauma $\left(7.1 \%{ }^{25}\right)$ with the occurrence of childhood trauma in the present sample. Pearson's correlations were computed to determine associations between symptoms of dissociation (continuous variable), the sum of childhood and adulthood trauma (continuous variables), and participants' age. Further, visual inspections of scatter plots revealed non-linear associations between dissociation and adulthood trauma, and between age and adulthood trauma. Accordingly, two non-linear correlation computations were performed.

Associations between the categories of dissociation (no dissociation; some experiences of dissociation; elevated experiences of dissociation; clearly elevated experiences of dissociation; high possibility of dissociative identity disorder) and the occurrence of childhood (yes vs no) and adulthood (yes vs no) trauma were first determined by an ordinal logistic regression model and afterwards with two independent Chi-square tests. Next, with a multiple regression analysis we examined whether the adulthood trauma (continuous variable) could be predicted as a function of sociodemographic information (age, marital status, highest educational level), childhood trauma and the degree of dissociation (continuous variables).

The nominal level of significance was set at alpha $\leq$ 0.05. All statistical computations were performed with SPSS $^{\circledR} 25.0$ (IBM Corporation, Armonk NY, USA) for Apple $\mathrm{Mac}^{\circledR}$.

\section{Results}

\section{Sociodemographic Results}

Table 1 provides the sociodemographic characteristics of participants.

A total of 69 male incarcerated people (mean age: 33.76 years; $\mathrm{SD}=5.74$; range: $25-48$ years) with borderline personality disorder participated in the present study. The majority were either single or married. In addition, 56 $(81.2 \%)$ reported at least one type of childhood abuse, while $13(18.8 \%)$ reported no childhood abuse. Similarly, $61(88.4 \%)$ participants reported a current/adult traumatic event, while 8 (11.6\%) did not.
Table I Frequency and Percentage of Demographic Characteristics, Dissociative Symptoms, Childhood Traumas, and Adulthood Traumas in Male Prisoners with Borderline Personality Disorder $(\mathrm{N}=69)$

\begin{tabular}{|c|c|c|}
\hline \multirow[t]{2}{*}{ Dimension } & \multicolumn{2}{|l|}{$M(S D)$} \\
\hline & $\mathbf{N}$ & $\%$ \\
\hline Age (years) & $33.72(5.74)$ & \\
\hline \multicolumn{3}{|l|}{ Educational level } \\
\hline Illiterate and elementary school & 7 & 10.1 \\
\hline Guidance $^{\mathrm{a}}$ and high school & 43 & 62.3 \\
\hline Academic level & 19 & 27.5 \\
\hline \multicolumn{3}{|l|}{ Current job position } \\
\hline Jobless & 21 & 30.4 \\
\hline Self-employment & 48 & 69.6 \\
\hline \multicolumn{3}{|l|}{ Marital status } \\
\hline Single & 32 & 46.4 \\
\hline Married & 33 & 47.8 \\
\hline Divorced & 4 & 5.8 \\
\hline \multicolumn{3}{|l|}{ Living area } \\
\hline Urban areas & 62 & 89 \\
\hline Rural areas & 7 & 10.1 \\
\hline \multicolumn{3}{|l|}{ Childhood trauma } \\
\hline No reported childhood trauma & 13 & 18.8 \\
\hline At least one reported childhood trauma & 17 & 24.6 \\
\hline At least two reported childhood traumas & 16 & 23.2 \\
\hline At least three reported childhood traumas & 16 & 23.2 \\
\hline At least four reported childhood traumas & 4 & 5.8 \\
\hline At least five reported childhood traumas & 1 & 1.4 \\
\hline At least six reported childhood traumas & 2 & 2.9 \\
\hline \multicolumn{3}{|l|}{ Adulthood trauma } \\
\hline No reported adulthood trauma & 8 & 11.6 \\
\hline At least one reported adulthood trauma & 13 & 18.8 \\
\hline At least two reported adulthood traumas & 12 & 17.4 \\
\hline At least three reported adulthood traumas & 11 & 15.9 \\
\hline At least four reported adulthood traumas & 14 & 20.3 \\
\hline At least five reported adulthood traumas & 6 & 8.7 \\
\hline At least six reported adulthood traumas & 4 & 5.8 \\
\hline Seven reported adulthood traumas & I & 1.4 \\
\hline \multicolumn{3}{|l|}{ Dissociative disorders } \\
\hline Yes & 23 & 33.3 \\
\hline No & 46 & 66.7 \\
\hline \multicolumn{3}{|l|}{ Type of crime committed } \\
\hline Theft & 38 & 55.1 \\
\hline Dispute & 6 & 8.7 \\
\hline Drug dealing & 20 & 29 \\
\hline Kidnapping & 2 & 2.9 \\
\hline Gambling & I & 1.4 \\
\hline Financial cheating & 1 & 1.4 \\
\hline Familial issues & 1 & 1.4 \\
\hline
\end{tabular}

Notes: ${ }^{\text {a }}$ guidance school $=$ most modest level of mandatory secondary school. 


\section{Occurrence of Childhood Trauma (\%), Compared to Normative Data}

The occurrence of childhood trauma was $81.2 \%$, which was significantly higher than the value for normative data $(7.1 \% ; \mathrm{t}(68)=109.41, \mathrm{p}<0.001)$.

\section{Childhood and Current Adulthood}

\section{Trauma and Dissociation}

Table 2 reports the descriptive statistical indices and the correlation coefficients (which are not repeated in the text anymore). Symptoms of dissociation were statistically unrelated to childhood trauma, to adulthood trauma and to age. Higher childhood trauma was statistically significantly associated with adulthood trauma, but not with age. Higher adulthood trauma was associated with younger age.

A visual inspection of the scatter plot between scores of dissociation and adulthood trauma showed that the linear correlation obscured the more complex association, while a non-linear and U-shaped computation was more precise: both lower (0-2) and higher adulthood trauma scores (5-7) were associated with higher scores of dissociation $\left(\mathrm{R}^{2}=0.281, \mathrm{r}=0.53, \mathrm{p}<0.001\right)$.

Next, a further visual inspection of the scatter plot between age and adulthood trauma showed that the negative linear correlation $(\mathrm{r}=-.28)$ obscured the more complex correlation, while a non-linear computation showed a correlation coefficient of $\mathrm{r}=0.47\left(\mathrm{p}<0.001 ; \mathrm{R}^{2}=0.22\right)$. More specifically, the U-shaped curve showed that both lower (0-2) and higher adulthood trauma scores (5-7) were associated with higher age, while 2-5 scores were associated with lower age.

Next, to predict the categorical variables of dissociation (no dissociation; some experiences of dissociation; elevated experiences of dissociation; clearly elevated experiences of dissociation; high possibility of dissociative identity disorder), and childhood trauma (yes/no) and adulthood trauma (yes/no) an ordinal logistic regression model was performed. The model fit was $\mathrm{X}^{2}(\mathrm{~N}=69, d f$ $=2)=3.35, \mathrm{p}=19$; or in other words: the predictors (childhood and adulthood traumas) could not statistically sufficiently explain the degree of dissociation. Given this, two separate X2-tests were performed. Table 3 reports the associations of categorical variables of dissociation (no dissociation; some experiences of dissociation; elevated experiences of dissociation; clearly elevated experiences of dissociation; high possibility of dissociative identity disorder), and childhood trauma (yes/no) and adulthood trauma (yes/no). The presence of childhood trauma was associated with a higher category of dissociation; the presence of adulthood trauma was not associated with a higher category of dissociation (statistical indices in Table 3).

\section{Predicting Current/Adulthood Trauma}

Table 4 reports the statistical indices of the multiple regression analysis with current/adulthood trauma as outcome variable and sociodemographic characteristics and childhood trauma and the degree of dissociation (always as continuous variables) as predictors.

Higher childhood trauma scores predicted higher current/adulthood trauma scores; descriptively, lower age, and being single or divorced also predicted higher current/ adulthood traumas, while level of education and degree of dissociation (continuous dimension) were not significant predictors $(\mathrm{p}>0.05)$ and were excluded from the equation.

\section{Discussion}

The key findings of the present study were that, among a small sample of adult Iranian male incarcerated people with borderline personality disorders (BPD), retrospectively self-reported childhood trauma was related to current/adulthood trauma. Furthermore, compared to

Table 2 Descriptive Statistical Indices and Correlation Coefficients of Symptoms of Dissociation, Childhood Trauma, Adulthood Trauma and Age (Always Continuous Variables)

\begin{tabular}{|l|l|l|l|l|l|}
\hline & \multirow{2}{*}{$\begin{array}{l}\text { Symptoms of } \\
\text { Dissociation }\end{array}$} & Dimensions & Adulthood Trauma & \multirow{2}{*}{ Age } & Descriptive Statistics \\
\cline { 3 - 5 } & Childhood Trauma & & & M (SD) \\
\hline Symptoms of dissociation & - & 0.18 & 0.03 & -0.22 & $38.36(17.33)$ \\
Childhood trauma & & - & $0.39 * *$ & -0.18 & $1.88(1.44)$ \\
Adulthood trauma & & & - & $-0.28^{*}$ & $2.71(1.38)$ \\
Age (years) & & & - & $33.72(5.74)$ \\
\hline
\end{tabular}

Notes: ${ }^{*} p<0.05 ; *^{*} p<0.01$. 
Table 3 Occurrence of Dissociative Symptoms and the Occurrence of Childhood and Adulthood Trauma in Male Prisoners with Borderline Personality Disorder

\begin{tabular}{|c|c|c|c|c|c|c|c|c|}
\hline & & & & $\begin{array}{l}\text { Categories of } \\
\text { Dissociation }\end{array}$ & & & & \multirow[t]{3}{*}{ Statistics } \\
\hline & & & $\begin{array}{l}\text { No } \\
\text { dissociation }\end{array}$ & $\begin{array}{l}\text { Some } \\
\text { experiences }\end{array}$ & $\begin{array}{l}\text { Elevated } \\
\text { experiences }\end{array}$ & $\begin{array}{l}\text { Clearly elevated } \\
\text { experiences }\end{array}$ & $\begin{array}{l}\text { High } \\
\text { possibility of } \\
\text { DID }\end{array}$ & \\
\hline & & $n(\%$ of 69$)$ & $n(\%$ of 69$)$ & $n(\%$ of 69$)$ & $n(\%$ of 69$)$ & $n(\%$ of 69$)$ & $n(\%$ of 69$)$ & \\
\hline $\begin{array}{l}\text { Childhood } \\
\text { trauma }\end{array}$ & $\begin{array}{l}\text { Yes } \\
\text { No }\end{array}$ & $\begin{array}{l}56(81.2) \\
13(18.8)\end{array}$ & $\begin{array}{l}7(10.1) \\
3(4.3)\end{array}$ & $\begin{array}{l}5(7.2) \\
I(1.4)\end{array}$ & $\begin{array}{l}3(4.3) \\
4(5.8)\end{array}$ & $\begin{array}{l}9(13.0) \\
I(1.4)\end{array}$ & $\begin{array}{l}32(46.4) \\
4(5.8)\end{array}$ & $\begin{array}{l}X^{2}(N=69, d f \\
=4)=9.47^{*}\end{array}$ \\
\hline $\begin{array}{l}\text { Adulthood } \\
\text { trauma }\end{array}$ & $\begin{array}{l}\text { Yes } \\
\text { No }\end{array}$ & $\begin{array}{l}61(88.4) \\
8(11.6)\end{array}$ & $\begin{array}{l}9(13.0) \\
1(1.4)\end{array}$ & $\begin{array}{l}5(7.2) \\
I(1.4)\end{array}$ & $\begin{array}{l}5(7.2) \\
2(2.9)\end{array}$ & $\begin{array}{l}2(2.9) \\
0(0.0)\end{array}$ & $\begin{array}{l}32(32.4) \\
4(5.8)\end{array}$ & $\begin{array}{l}X^{2}(N=69, d f \\
=4)=3.46 \mathrm{~ns}\end{array}$ \\
\hline
\end{tabular}

Notes: ${ }^{*} \mathrm{p}<0.05$.

Abbreviations: DID, dissociative identity disorder; ns, not significant.

Table 4 Multiple Linear Regression with Current/Adulthood Trauma as Outcome Variable, and Childhood Trauma, Dissociation and Sociodemographic Variables as Predictors

\begin{tabular}{|l|l|l|l|l|l|l|l|l|l|}
\hline Dimension & Variables & Coefficient & Standard Error & Coefficient $\boldsymbol{\beta}$ & $\mathbf{t}$ & $\mathbf{P}$ & $\mathbf{R}$ & $\mathbf{R}^{2}$ & Durbin-Watson \\
\hline \multirow{2}{*}{ Adulthood trauma } & Intercept & 2.97 & 1.83 & - & 1.62 & $0.1 \mathrm{I}$ & 0.49 & 0.24 & 2.08 \\
& Childhood trauma & 0.49 & 0.14 & 0.39 & $3.4 \mathrm{I}$ & 0.01 & & & \\
& Age & -0.07 & 0.04 & -0.22 & 1.94 & 0.06 & & \\
& DES scores & -0.00 & 0.01 & -0.04 & 0.33 & 0.75 & & \\
& Marital status $^{2}$ & 0.06 & 0.35 & 0.19 & 1.69 & 0.09 & & \\
& Educational level $^{3}$ & 0.10 & 0.19 & 0.06 & 0.55 & 0.59 & & & \\
\end{tabular}

Notes: continuous variable. ${ }^{2}$ categorization of marital status: $\mathrm{I}=$ married; 2 = single; $3=$ divorced. $^{3}$ categorization of educational levels: $\mathrm{I}=$ illiterate and elementary school; 2 = guidance and high school; 2 = academic level.

Abbreviation: DES, Dissociative Experience Scale.

normative data, the childhood trauma rates for participants were significantly higher. Next, current/adulthood trauma was predicted by lower age, the occurrence of childhood trauma, and being single or divorced, while symptoms of dissociation were not related. Last and most importantly, two non-linear and U-shaped correlational computations showed that adulthood trauma and age and scores of dissociation were associated in a more complex fashion: Very low and very high scores of adulthood trauma were associated with older age and with higher scores of dissociation. The present results add to the current literature in an important way in showing a link (though moderate) between childhood trauma and adult trauma, in showing that younger, single or divorced male incarcerated people with borderline personality disorders were particularly likely to have higher scores for current/adulthood traumata, and above all in showing unexpected and novel non-linear associations between dissociation and adulthood trauma.
Two hypotheses and one research question were formulated and each of these is considered in turn.

Our first hypothesis was that prevalence rates for selfreported childhood trauma would be higher in male incarcerated people with BPD than the values for normative data, and this was confirmed. Accordingly, this result is consistent with findings previously reported in this field. ${ }^{17,19}$ However, with the present data, we expand upon previous findings in confirming this pattern among male incarcerated people in Iran, and in showing an association between childhood trauma and current trauma.

An issue appears to be the characteristics of normative data. While we relied on a recently published paper, ${ }^{25}$ aSR and MA in this field in Iran reported estimated prevalence rates for, respectively, childhood physical and emotional abuse of $43.6 \%$ and $64.5 \% .{ }^{33}$ Currently, we have no explanation as to why prevalence rates differ between studies. It is possible that assessment tools, 
respondents (parents; teachers; children), anonymity guarantees and other unknown factors might have contributed to the large discrepancies in prevalence rates reported in previous studies. However, the key concern was to compare participants' remembered childhood trauma with current childhood trauma prevalence rates as "normative data". To this end, the following key criteria were established: 1. Most recent data; 2. Original data from Iran; 3. No meta-analyses or meta-regressions, as such analyses may obscure and bias more fine-grained patterns of results; 4. Data were gathered anonymously to avoid social constraints; 5. Children completed the questionnaires; 6. Use of an appropriate self-rating tool to assess childhood trauma. The publication of Shamohammadi et $\mathrm{al}^{25}$ satisfied all key criteria: Shamohammadi et $\mathrm{al}^{25}$ run their study in 2018 and published the data in 2019 (criteria 1 and 3); they assessed a sample of Iranian 608 children (mean age: 11.5 years; $51.5 \%$ females) (criteria 2 and 5); participants completed anonymously the Trauma Symptoms Checklist for Children (criteria 4 and 6). Given this background, the decision to rely on the publication of Shamohammadi et $\mathrm{al}^{25}$ was very well considered.

Our second hypothesis anticipated an association between levels of dissociation and the prevalence rates for self-reported childhood and current/adulthood trauma. At a first glance, this assumption was not fully supported: Although higher scores for childhood trauma were associated with dimensions of current dissociation, the dimensions of current dissociation were apparently not associated with current/adulthood trauma. This finding deserves particular attention in that it appears that dissociation, which is to say the splitting of psychological processes such as perception, memory, emotion regulation, consciousness and identity, was not related to currently experienced traumas but to traumas experienced in childhood (and adolescence). This pattern of results is at odds with previously reported findings. ${ }^{19,22,23}$ Briere $^{34}$ observed that among the general population only $8 \%$ of those experiencing childhood trauma also reported symptoms of dissociation. On the other hand, nine out of ten individuals who reported two or more symptoms of dissociation also reported traumatic events in childhood and adolescence. Briere ${ }^{34}$ concluded that traumatic experiences in the past are important but not by themselves a sufficient factor to trigger symptoms of dissociation and that other factors such as current critical life events, posttraumatic stress, and poor emotion regulation skills might be among the significant additional conditions needed to trigger and maintain symptoms of dissociation.

However, at a second glance, the linear correlational computation $(\mathrm{r}=0.03)$ obscured the non-linear and U-shaped association between dissociation and adulthood trauma $(\mathrm{r}=0.53)$. Both low and high adulthood trauma scores were associated with higher scores of dissociation, while medium adulthood scores were associated with lower scores of dissociation. The quality of the data does not allow a deeper understanding of such kind of non-linear association. Though speculative, the following assumptions are made: 1 . It is plausible that participants reporting high adult trauma also report high scores of dissociation. ${ }^{19,21,23,35} 2$. By definition, dissociation is a coping strategy to deal with particularly threatening and unbearable situations. ${ }^{19,21,23,35}$ It is therefore conceivable that individuals experiencing recently particularly threatening situations are aware to dissociate, while the trigger of such dissociations remains obscured, and accordingly not reported in the questionnaires. 3. Relatedly, participants experiencing very recently traumatic events developed very quickly sings of dissociation; we were unable to test this hypothesis with the present data, and a literature search did not answer to the question of how much time it needs to develop signs of dissociation after a traumatic event. 4. Relatedly, being in prison and experiencing prison life might be a traumatic event per se; it is therefore conceivable that participants being in prison for the first time might have been at particular risk to develop quickly signs of dissociation. 5. Symptoms of dissociation are not fully related to current/adulthood trauma, but to childhood trauma (as shown in previous computations). 6. The pattern of results is an epiphenomenon of latent, but unassessed dimensions, which might have biased both scores of dissociation and scores of adulthood trauma in the same and in opposite directions. In this view, 7., Leichsenring et $\mathrm{al}^{12}$ mentioned that the emergence of BPD is related to genetic, environmental, and the gene-environment interactions. It is therefore possible that participants with a framework of particularly disadvantaged gene-environment-interaction were also at increased risk to suffer from dissociations. 8 . The questionnaire on current/adulthood trauma was too coarse-grained and did not ask of specific traumas, which occurred very recently and/or in prison.

Next, the visual inspection of the association between age and adulthood trauma showed that the linear correlation obscured the non-linear and U-shaped association: Both low and high adulthood trauma scores were associated with older age, while medium adulthood scores 
were observed among younger participants. Again, the quality of the data does not allow a deeper understanding of such kind of non-linear association; while intuitively it appears reasonable that older participants had a longer life time and thus more "chances" to experience more traumas as adults, this assumption is at odds with the opposite pattern of results: Older participants also reported very low scores of adulthood trauma. It is possible that the present pattern of results might be explained by further latent, but unassessed psychological variables which biased dimensions of adulthood trauma in opposite directions. While no definite answers could be given to these possibilities, future studies should carefully check their data for non-linear biases.

Our exploratory research question concerned the extent to which self-reported current trauma could be predicted. It turned out that the strongest predictors were the occurrence of childhood traumas and younger age, while descriptively being single or divorced was an additional predictor. Education, and dimensions of dissociations were not predictors. To turn another way around: Younger and single male incarcerated people with BPD and childhood trauma were at risk to suffer also from adulthood trauma, while a higher education was not a protective factor.

In the present study, the prevalence rate for dissociation was $33.3 \%$ (23 individuals), which is descriptively higher than the $10 \%$ value for college students, ${ }^{36}$ and descriptively higher than the prevalence rate of $3.4 \%$ for the general population in Kashan. ${ }^{37}$ Sar et al ${ }^{35}$ have reported prevalence rates of around $10 \%$ for dissociative disorders among inpatients and outpatients with psychiatric issues. In contrast, the prevalence rates in the present study were lower than those found for individuals with BPD; Brand and Lanius ${ }^{38}$ reported that $30 \%$ to $70 \%$ of patients with dissociative disorders also showed symptoms of BPD, while on the other hand, $41 \%$ to $72 \%$ of individuals with BPD are diagnosed with a dissociative disorder.

Despite the novelty of the results, the following limitations warrant against over-generalization. First, the sample size was rather small, and a larger sample might have yielded other meaningful and significant results. Second, inclusion and exclusion criteria were such that only a restricted sample of male incarcerated people were assessed; thus, the results do not generalize to female incarcerated people, or to male incarcerated people with other than a diagnosis of BPD. In the same vein, a sample with "pure" participants with BPD and without further comorbidities could bear the risk to poorly reflect the clinical reality of individuals with BPD and a broad range of concomitant psychiatric issues. While thus the sample might be biased and poorly reflect what is daily seen in a prison, on the flip side, psychiatric issues such as substance use disorder, major depressive disorders, anxiety disorders or impulse control disorders could be ruled out to confound and bias the present pattern of results. Third, intake of psychotropic medications was not assessed, though it is entirely possible that sedative/hypnotics negatively impacted on participants' memory and concentration. Fourth, by nature, not all psychological and psychophysiological dimensions were assessed; it follows that further latent and unassessed psychological factors might have biased the present pattern of results. This holds particularly true, as both Favril et $\mathrm{al}^{10}$ and Verdolini et $\mathrm{al}^{11}$ showed that BPD, along with misuse of multiple substances and major depressive disorders, was highly associated with self-harming behavior. Given this, future studies should consider self-harming behavior as an important behavior-moderating factor. Fifth, the Traumatic Experience Checklist (TEC ${ }^{31,32}$ ) includes incarceration as a traumatic event during adulthood. While the decision was to keep the set of items of the questionnaire unaltered to avoid the risk of invalidating psychometrically the questionnaire, it is conceivable that this redundancy might have yielded a biased pattern of results.

\section{Conclusion}

Among a smaller sample of male Iranian incarcerated people with borderline personality disorders high rates of self-reported childhood and adulthood trauma were observed. Trauma and dissociation are not necessarily and above all not linearly associated. Linear correlational computations might obscure more complex associations between trauma and dissociation. Further studies should consider non-linear associations.

\section{Acknowledgment}

The authors wish to thank all those people who took part in this study. This paper is based on the thesis of Sahel Ghanbari (Kermanshah University of Medical Sciences, Kermanshah, Iran; 95462), submitted to the School of Medicine in partial fulfillment of the degree requirements for general physician. We also thank Nick Emler (University of Surrey, Surrey UK) for proofreading the manuscript. 


\section{Disclosure}

The authors have no conflicts of interest to disclose for this work. Sanobar Golshani and Sahel Ghanbari shared first authorship.

\section{References}

1. Fazel S, Chang Z, Fanshawe T, et al. Prediction of violent reoffending on release from prison: derivation and external validation of a scalable tool. Lancet Psychiatry. 2016;3(6):535-543. doi:10.1016/ S2215-0366(16)00103-6

2. Ahmed R, Angel C, Martel R, Pyne D, Keenan L. Access to healthcare services during incarceration among female inmates. Int J Prison Health. 2016;12(4):204-215. doi:10.1108/IJPH-04-2016-0009

3. Baranyi G, Scholl C, Fazel S, Patel V, Priebe S, Mundt AP. Severe mental illness and substance use disorders in prisoners in low-income and middle-income countries: a systematic review and meta-analysis of prevalence studies. Lancet Global Health. 2019;7(4):e461-e471. doi:10.1016/S2214-109X(18)30539-4

4. Fazel S, Hayes AJ, Bartellas K, Clerici M, Trestman R. Mental health of prisoners: prevalence, adverse outcomes, and interventions. Lancet Psychiatry. 2016;3(9):871-881. doi:10.1016/S2215-0366(16)30142-0

5. Henry BF. Typologies of adversity in childhood \& adulthood as determinants of mental health \& substance use disorders of adults incarcerated in US prisons. Child Abuse Negl. 2020;99:104251. doi:10.1016/j.chiabu.2019.104251

6. Conn C, Warden R, Stuewig J, et al. Borderline personality disorder among jail inmates: how common and how distinct? Corrections Compendium. 2010;35(4):6-13.

7. Reingle Gonzalez JM, Connell NM. Mental health of prisoners: identifying barriers to mental health treatment and medication continuity. Am J Public Health. 2014;104(12):2328-2333.

8. Marzano L, Hawton K, Rivlin A, Smith EN, Piper M, Fazel S. Prevention of suicidal behavior in prisons. Crisis. 2016;37 (5):323-334. doi:10.1027/0227-5910/a000394

9. Valizadeh R, Veisani Y, Delpisheh A, Kikhavani S, Sohrabnejad A. Major depression and psychiatric disorders in Iranian prisoners based on a clinical interview: a systematic review and meta-analysis. Shiraz E-Med J. 2017;18(6):e44979.

10. Favril L, Yu R, Hawton K, Fazel S. Risk factors for self-harm in prison: a systematic review and meta-analysis. Lancet Psychiatry. 2020;7(8):682-691. doi:10.1016/S2215-0366(20)30190-5

11. Verdolini N, Murru A, Attademo L, et al. The aggressor at the mirror: psychiatric correlates of deliberate self-harm in male prison inmates. Eur Psychiatry. 2017;44:153-160. doi:10.1016/j.eurpsy.20 17.04 .002

12. Leichsenring F, Leibing E, Kruse J, New AS, Leweke F. Borderline personality disorder. Lancet (London, England). 2011;377 (9759):74-84. doi:10.1016/S0140-6736(10)61422-5

13. Sepehrmanesh Z, Ahmadvand A, Akasheh G, Saei R. Prevalence of psychiatric disorders and related factors in male prisoners. Iran Red Crescent Med J. 2014;16(1):e15205. doi:10.5812/ircmj.15205

14. Giedd JN, Blumenthal J, Jeffries NO, et al. Brain development during childhood and adolescence: a longitudinal MRI study. Nat Neurosci. 1999;2(10):861-863. doi:10.1038/13158

15. Paus T, Keshavan M, Giedd JN. Why do many psychiatric disorders emerge during adolescence? Nat Rev Neurosci. 2008;9(12):947-957. doi: $10.1038 / \mathrm{nrn} 2513$

16. Mohr-Jensen C, Steinhausen HC. A meta-analysis and systematic review of the risks associated with childhood attention-deficit hyperactivity disorder on long-term outcome of arrests, convictions, and incarcerations. Clin Psychol Rev. 2016;48:32-42. doi:10.1016/j. cpr.2016.05.002
17. Hock RS, Bryce CP, Fischer L, et al. Childhood malnutrition and maltreatment are linked with personality disorder symptoms in adulthood: results from a Barbados lifespan cohort. Psychiatry Res. 2018;269:301-308. doi:10.1016/j.psychres.2018.05.085

18. Konkoly Thege B, Horwood L, Slater L, Tan MC, Hodgins DC, Wild TC. Relationship between interpersonal trauma exposure and addictive behaviors: a systematic review. BMC Psychiatry. 2017;17 (1):164. doi:10.1186/s12888-017-1323-1

19. Vermetten E, Spiegel D. Trauma and dissociation: implications for borderline personality disorder. Curr Psychiatry Rep. 2014;16 (2):434. doi:10.1007/s11920-013-0434-8

20. American Psychiatric Association. Diagnostic and Statistical Manual of Mental Disorders 5th Edition: DSM 5. Arlington VA: American Psychiatric Association; 2013.

21. Spiegel D, Lewis-Fernández R, Lanius R, Vermetten E, Simeon D, Friedman M. Dissociative disorders in DSM-5. Annu Rev Clin Psychol. 2013;9:299-326. doi:10.1146/annurev-clinpsy-050212-185531

22. Scalabrini A, Cavicchioli M, Fossati A, Maffei C. The extent of dissociation in borderline personality disorder: a meta-analytic review. J Trauma Dissoc. 2017;18(4):522-543.

23. Sar V, Alioglu F, Akyuz G. Depersonalization and derealization in self-report and clinical interview: the spectrum of borderline personality disorder, dissociative disorders, and healthy controls. J Trauma Dissoc. 2017;18(4):490-506.

24. Tyrka AR, Wyche MC, Kelly MM, Price LH, Carpenter LL. Childhood maltreatment and adult personality disorder symptoms: influence of maltreatment type. Psychiatry Res. 2009;165 (3):281-287. doi:10.1016/j.psychres.2007.10.017

25. Shamohammadi M, Salmanian M, Mohammadi MR, Sadeghi Bahmani D, Holsboer-Trachsler E, Brand S. Prevalence of selfreported trauma in a sample of Iranian children is low and unrelated to parents' education or current employment status. Revista Brasileira de Psiquiatria (Sao Paulo, Brazil: 1999). 2019;41 (3):208-212. doi:10.1590/1516-4446-2018-0146

26. World Medical Association. World Medical Association Declaration of Helsinki: ethical principles for medical research involving human subjects. JAMA. 2013;310(20):2191-2194. doi:10.1001/jama.2013.281053

27. First MB, Spitzer RL, Gibbon M, Williams JBW. The structured clinical interview for DSM-III-R Personality Disorders (SCID-II). Part I: description. J Pers Disord. 1995;9(2):83-91. doi:10.1521/ pedi.1995.9.2.83

28. Sheehan DV, Lecrubier Y, Sheehan KH, et al. The Mini-International Neuropsychiatric Interview (M.I.N.I.): the development and validation of a structured diagnostic psychiatric interview for DSM-IV and ICD-10. J Clin Psychiatry. 1998;59(Suppl 20):22-33;quiz34-57.

29. Bernstein EM, Putnam FW. Development, reliability, and validity of a dissociation scale. J Nerv Ment Dis. 1986;174(12):727-735. doi:10.1097/00005053-198612000-00004

30. Ghaffarinejad A, Sattari N, Raaii F, Arjmand S. Validity and reliability of a Persian version of the Dissociative Experiences Scale II (DES-II) on Iranian patients diagnosed with schizophrenia and mood disorders. J Trauma Dissoc. 2019;1-12.

31. Sadeghi S, Dolatshahi B, Pourshahbaz A, Zarei M, Kami M. Relationship between traumatic experiences and somatic symptoms severity in students. Pract Clin Psychol. 2017;5(3):211-216. doi:10.18869/acadpub.jpcp.5.3.211

32. Nijenhuis ERS, Van der Hart O, Kruger K. The psychometric characteristics of the traumatic experiences checklist (TEC): first findings among psychiatric outpatients. Clin Psychol Psychother. 2002;9 (3):200-210. doi:10.1002/cpp.332

33. Mohammadi MR, Zarafshan H, Khaleghi A. Child abuse in Iran: a systematic review and meta-analysis. Iran J Psychiatry. 2014;9 (3):118-124.

34. Briere J. Dissociative symptoms and trauma exposure: specificity, affect dysregulation, and posttraumatic stress. J Nerv Ment Dis. 2006;194(2):78-82. doi:10.1097/01.nmd.0000198139.47371.54 
35. Sar V, Onder C, Kilincaslan A, Zoroglu SS, Alyanak B. Dissociative identity disorder among adolescents: prevalence in a university psychiatric outpatient unit. J Trauma Dissoc. 2014;15(4):402-419. doi:10.1080/15299732.2013.864748

36. Kate MA, Hopwood T, Jamieson G. The prevalence of dissociative disorders and dissociative experiences in college populations: a meta-analysis of 98 studies. $J$ Trauma Dissoc. 2019;1-46.
37. Ahmadvand A, Sepehrmanesh Z, Ghoreishi FS, Afshinmajd S. Prevalence of psychiatric disorders in the general population of Kashan, Iran. Arch Iran Med. 2012;15(4):205-209.

38. Brand BL, Lanius RA. Chronic complex dissociative disorders and borderline personality disorder: disorders of emotion dysregulation? Borderline Personal Disord Emot Dysregul. 2014;1:13. doi:10.1186/ 2051-6673-1-13

\section{Publish your work in this journal}

Neuropsychiatric Disease and Treatment is an international, peerreviewed journal of clinical therapeutics and pharmacology focusing on concise rapid reporting of clinical or pre-clinical studies on a range of neuropsychiatric and neurological disorders. This journal is indexed on PubMed Central, the 'PsycINFO' database and CAS, and is the official journal of The International Neuropsychiatric Association (INA). The manuscript management system is completely online and includes a very quick and fair peer-review system, which is all easy to use. Visit http://www.dovepress.com/testimonials.php to read real quotes from published authors.

Submit your manuscript here: https://www.dovepress.com/neuropsychiatric-disease-and-treatment-journal 